\title{
The Eigenvalues of Isolated Bridges with Transverse Restrains at the End Abutments
}

\author{
Nicos Makris ${ }^{1}$, Georgios Kampas ${ }^{2}$ and Dimitra Angelopoulou ${ }^{3}$ \\ Department of Civil Engineering, University of Patras, GR-26500, Greece
}

\begin{abstract}
SUMMARY
This paper examines the eigenvalues of multi-span seismically isolated bridges in which the transverse displacement of the deck at the end-abutments is restricted. With this constraint the deck is fully isolated along the longitudinal direction, while along the transverse direction the deck is a simple supported beam at the end abutments which enjoys concentrated restoring forces from the isolation bearings at the center piers. For moderate long bridges the first natural period of the bridge is the first longitudinal period, while the first transverse period is the second period, given that the flexural rigidity of the deck along the transverse direction shortens the isolation period offered by the bearings in that direction. This paper shows that for isolated bridges longer than a certain critical length, the first transverse period becomes longer than the first longitudinal period despite the presence of the flexural rigidity of the deck. This critical length depends on whether the bridge is isolated on elastomeric bearings or on spherical sliding bearings. This result is also predicted with established commercially available numerical codes only when several additional nodes are added along the beam elements which are modeling the deck in-between the bridge piers. On the other hand this result can not be captured with the limiting idealization of a beam on continuous distributed springs (beam on Wrinkler foundation) -a finding that has practical significance in design and system identification studies. Finally, the paper shows that the normalized transverse eigenperiods of any finite-span deck are selfsimilar solutions that can be represented by a single master curve and are independent of the longitudinal isolation period or on whether the deck is supported on elastomeric or spherical sliding bearings.

Keywords: Seismic Isolation; Bridge; Eigenvalues; System Identification; Self-Similarity; Dynamic Response

${ }^{1}$ Professor, Dept. of Civil Engineering, University of Patras, Greece, GR 26500

${ }^{2}$ Doctoral Candidate, Dept. of Civil Engineering, University of Patras, Greece, GR 26500

${ }^{3}$ Former Graduate Student, Dept. of Civil Engineering, University of Patras, Greece, GR 26500
\end{abstract}

\section{INTRODUCTION}

Seismic isolation, either with elastomeric or sliding bearings, is at present widely adopted as an effective technology for the seismic protection of highway and railway bridges (Skinner et al.[1], FHWA [2]). Traditionally, many conventionally designed bridges use elastomeric bearings (pads) between the deck and its supports to accommodate thermal movements. The long experience with this technology had a positive role on the implementation of modern seismic protection technologies in bridges (Kelly [3]).

The most commonly used isolation bearings are either elastomeric bearings or spherical sliding bearings. Figure 1 shows the view of two neighbor railway bridges currently under construction in central Greece. Both bridges are seismically isolated on identical spherical sliding bearings with radius of curvature $R=2.2 \mathrm{~m}$. The isolation period of the spherical bearings alone, in any horizontal direction is 

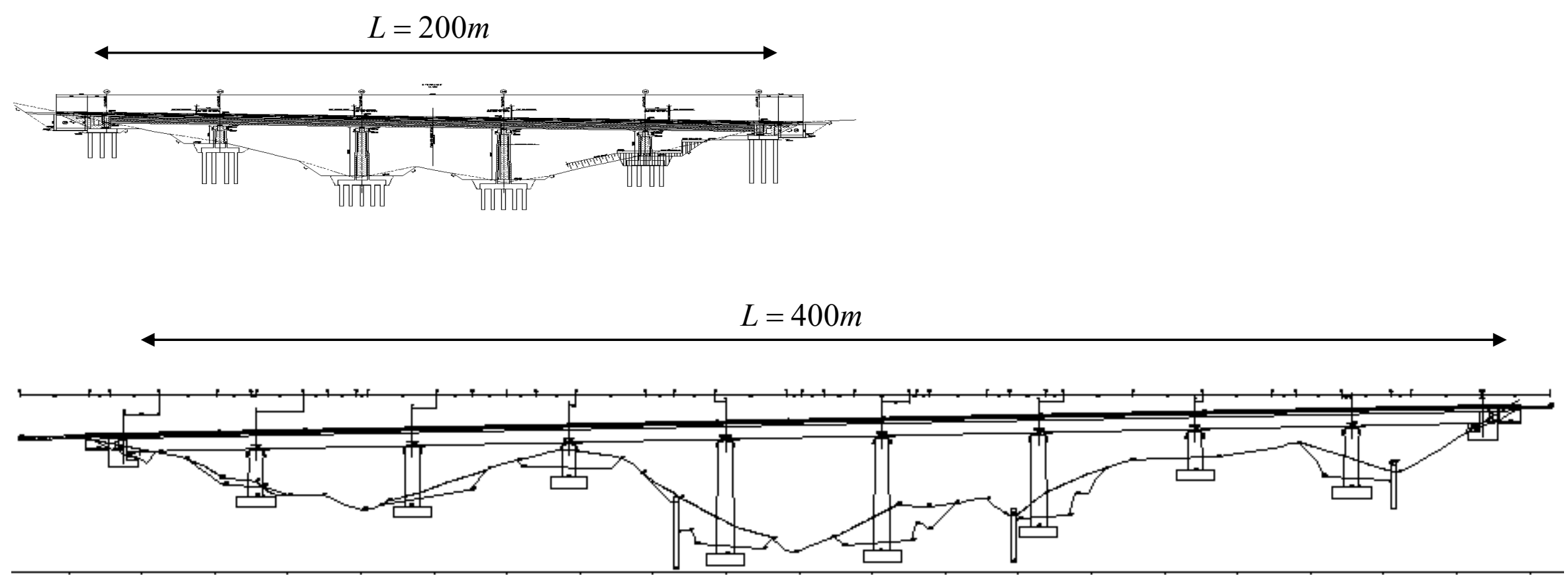

Figure 1. Elevation of two seismically isolated bridges. Top: 5-span 200m long bridge; Bottom: 9-span 400m long bridge 


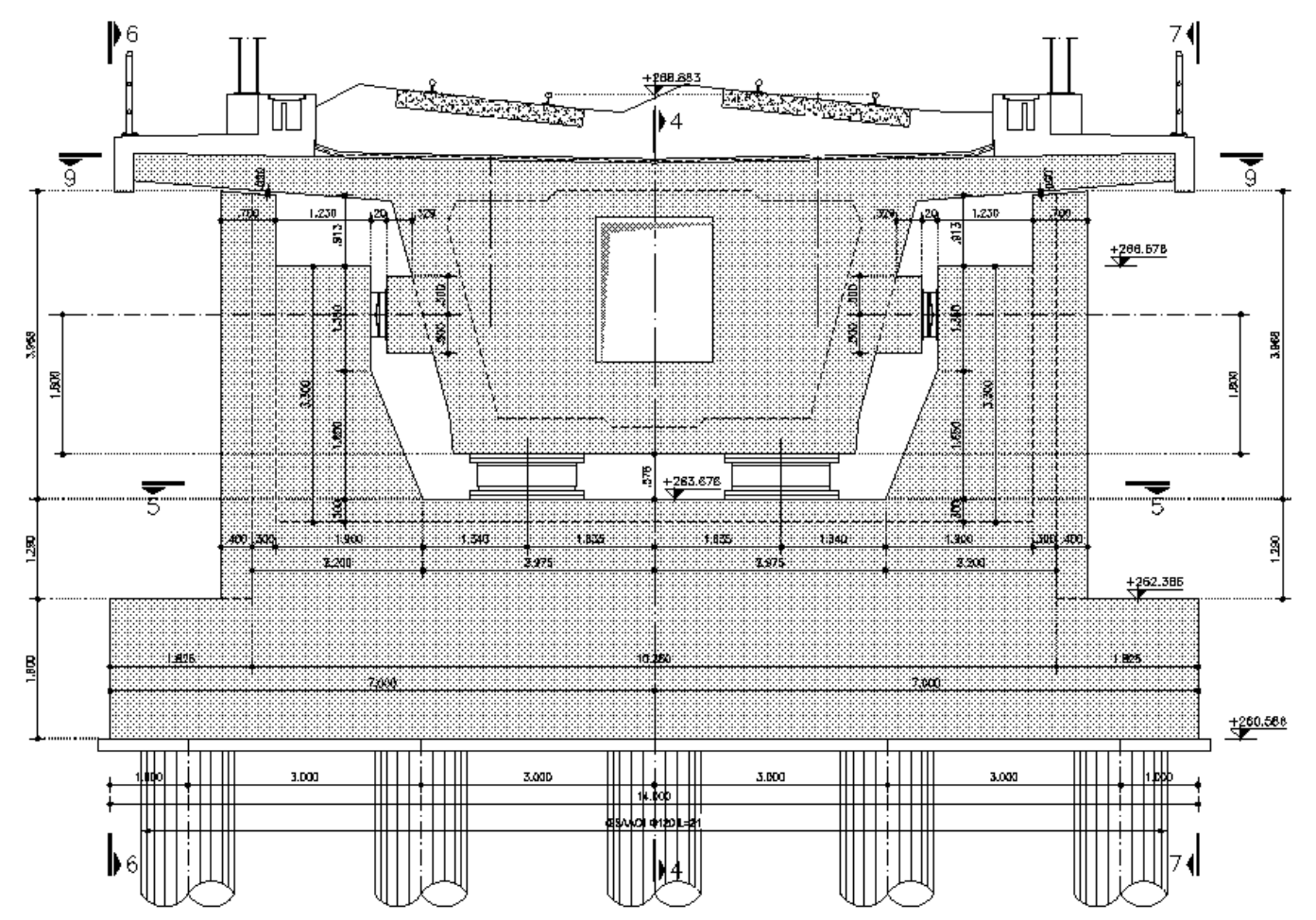

Figure 2. Cross section of the deck at the end-abutment which restrains the transverse motion of the deck.

$T_{I}=2 \pi \sqrt{R / g}=2.98 \mathrm{sec}$. Figure 2 shows a cross section of the deck at the endabutments of both bridges where the motion of the deck is restrained along the transverse direction. This restriction is nearly imperative in railway bridges in order to avoid misalignment of the rails at the deck-abutment joints during earthquake shaking; while, it is also common in highway bridges.

The eigenvalue analysis of both bridges can be conducted with a linear stick model using elastic beam elements. The stick model allows for the flexure of the center piers and the finite stiffness of pile foundations in the horizontal, vertical, rocking and cross horizontal- rocking directions (Zhang and Makris [3]).

Table 1 shows the eight first eigenvalues of the two seismically isolated bridges shown in Figure 1, for the case where $E_{p} / E_{s}=100$ ( $E_{p}=$ Young's modulus of pile $=25 \mathrm{GPa}$ for the concrete used, and $E_{s}=$ Young's modulus of soil). Table 1 shows that the five-span, $200 \mathrm{~m}$ long bridge has as first eigevalue the longitudinal, $T_{1}=T_{L}=3.07 \mathrm{sec}$, a value that is slightly larger than the spherical bearing period, $T_{I}=2 \pi \sqrt{R / g}=2.98 \mathrm{sec}$, due to the finite flexibility of piers and piles connected in series with the bearings. The second eigenvalue of the five-span, $200 \mathrm{~m}$-long, bridge is the first transverse eigenvalue, $T_{2}=T_{T}=2.17 \mathrm{sec}$, a value that is appreciably smaller from the spherical bearing period, $T_{I}=2 \pi \sqrt{R / g}=2.98 \mathrm{sec}$, due to the added transverse flexural rigidity of the deck which is simple-supported at the end-abutments (see Figure 2). 
Table 1 also shows that the longer nine-span, 400m long, bridge has as first eigenvalue, the first longitudinal eigenvalue, $T_{1}=T_{L}=3.16 \mathrm{sec}$ and very close, yet

Table 1. Eigenperiods of the 200m and 400m long isolated bridges shown in Figure 1.

\begin{tabular}{|c|c|c|}
\hline \multicolumn{3}{|c|}{ Eigenperiods $T(\mathrm{sec}), E_{p} / E_{s}=100$} \\
\hline Modes & 200m long bridge & $400 \mathrm{~m}$ long bridge \\
\hline 1 & 3.07 & 3.16 \\
\hline 2 & 2.17 & 3.13 \\
\hline 3 & 0.77 & 2.29 \\
\hline 4 & 0.36 & 1.38 \\
\hline 5 & 0.34 & 0.84 \\
\hline 6 & 0.28 & 0.71 \\
\hline 7 & 0.23 & 0.71 \\
\hline 8 & 0.22 & 0.571 \\
\hline
\end{tabular}

second eigenvalue, the transverse eigenvalue, $T_{2}=T_{T}=3.13 \mathrm{sec}$. This numerical result, where the transverse period has reached so closely the longitudinal period, was the main motivation for this study in order to examine whether it is possible that the transverse period may exceed the longitudinal period.

Our study proceeds with the mathematical analysis of the two limiting-case mechanical models: (a) that of a beam with transverse restrains at the end abutments supported with continuously distributing springs (beam on Wrinkler foundation); and (b) that of an isolated beam with transverse restrains at the end abutments and a single transverse and longitudinal spring at mid-span. The remarkable analytical result derived in this study -that beyond a certain length the two-span isolated bridge with transverse restrains at the end-abutments has a transverse period that exceeds the longitudinal period- is confirmed numerically with commercially available software for a 4-span and an 8-span bridge. The corresponding span lengths of the 4-span and 8 -span bridge that this phenomenon happens are technically realizable, therefore this finding has practical significance in design and system identification studies.

\section{MECHANICAL IDEALIZATION OF ISOLATED BRIDGES}

Figure 3 shows the mechanical idealization of the isolated bridge where the transverse motion of the deck is isolated with springs at the center piers and restrained at the end-abutments; while, the longitudinal motion is isolated with elastic springs everywhere. In order to capture the dynamic behavior of the mechanical configuration shown in Figure 3 we examine the mathematical solution of the two limiting cases that of a beam that is fully isolated along the longitudinal direction while restrained at the end abutments along the transverse direction and having either (a) infinite distributed transverse springs along the span (beam on Winkler supports) as shown in Figure 4, or (b) a single longitudinal and a single transverse spring at the mid-span as shown in Figure 5. 


\section{Elevation}

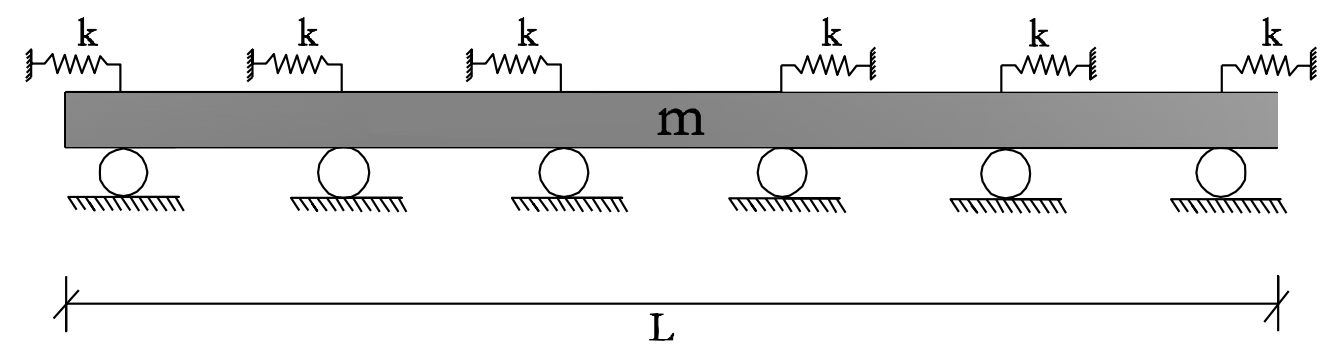

\section{Plan View}

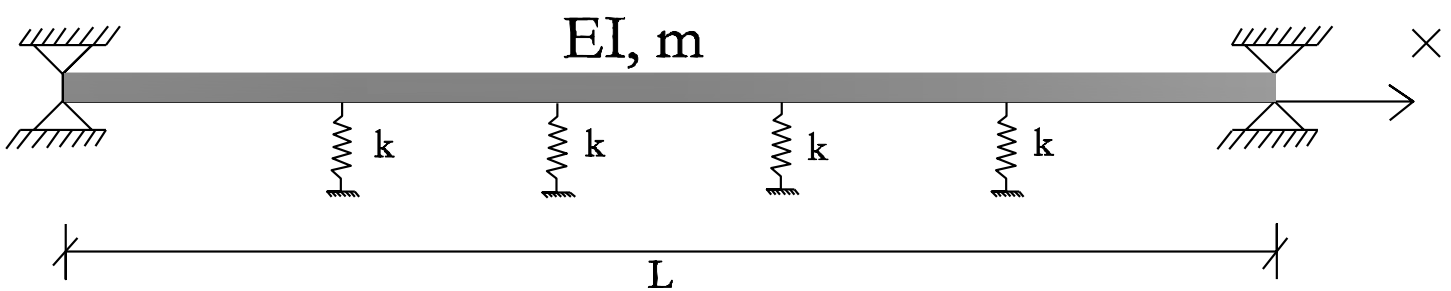

Figure 3. Mechanical idealization of an isolated deck which is fully isolated along the longitudinal direction while along the transverse direction the deck is isolated at the center supports and simple supported at the end-abutments.

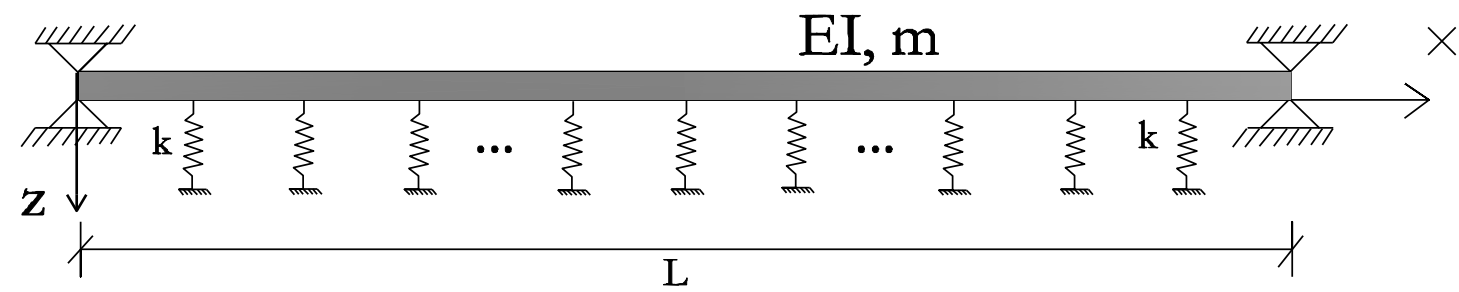

Figure 4. Plan view of a beam with continuous distributed springs along its length (Winkler foundation) that is simple supported along the transverse direction at its ends
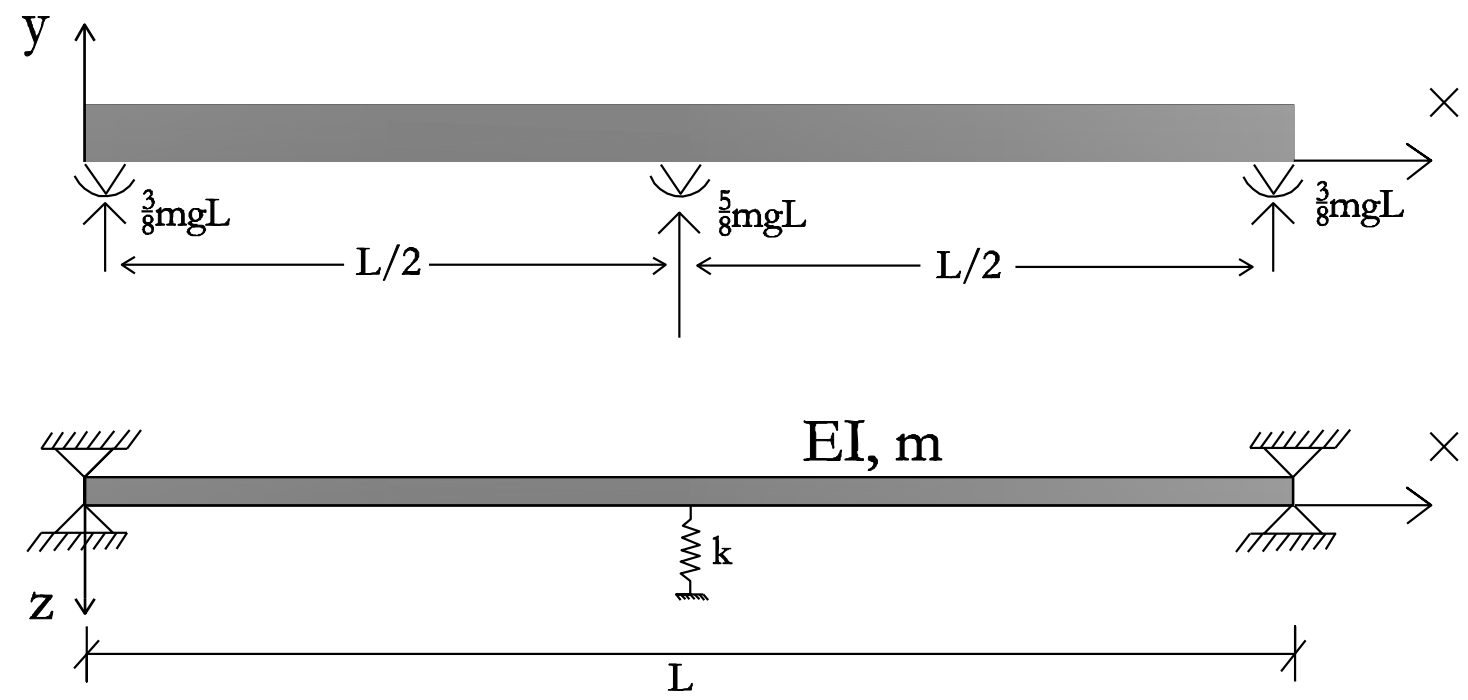

Figure 5.Top:Elevation of a two-span beam that is fully isolated along the longitudinal direction; Bottom: Plan view of the beam that is isolated at mid-span and is simple supported at its ends. 


\section{LONGITUDINAL AND TRANSVERSE EIGENVALUES OF A BEAM WITH CONTINUOUSLY DISTRIBUTED SPRINGS}

For a beam on continuously distributed elastic supports with stiffness $k[F] /[L]^{2}$ and distributed mass, $m[M] /[L]$; and assuming that the axial rigidity of the beam is large compared to its flexural rigidity, its first longitudinal eigenvalue is the isolation frequency along the longitudinal direction

$$
\omega_{L 1}=\omega_{I L}=\sqrt{\frac{k L}{m L}}=\sqrt{\frac{k}{m}}
$$

Under free vibration, the governing equation of motion along the transverse direction is (Timoshenko et al. [5], among others)

$$
E I \frac{d^{4} w(x)}{d x^{4}}+\left(k-m \omega^{2}\right) w(x)=0
$$

Case 0: $k-m \omega^{2}=0$

We examine whether the beam with distributed elastic supports may posses a transverse eigenfrequency equal to the longitudinal eigenfrequency $\omega=\omega_{I T}=\omega_{I L}=\sqrt{k / m}$. In this event, $k=m \omega^{2}$, and Equation (2) contracts to :

$$
E I \frac{d^{4} w(x)}{d x^{4}}=0
$$

The solution of Equation (3) is

$$
w(x)=A x^{3}+B x^{2}+C x+D
$$

The boundary conditions of this configuration for vibrations along the transverse direction are zero translation at the end-abutments $(w(0)=w(L)=0)$ and zero moments at the end-abutments $\left(\frac{d^{2} w(0)}{d z^{2}}=\frac{d^{2} w(L)}{d z^{2}}=0\right)$. Given that the second derivative of Equation (4) is

$$
\frac{d^{2} w(z)}{d z^{2}}=6 A x+2 B
$$

the condition $d^{2} w(0) / d z^{2}=0$ yields $B=0$; and upon enforcing the condition $\frac{d^{2} w(L)}{d z^{2}}=0, A=0$. Accordingly, $w(x)=C x+D$, and the application of the conditions $w(0)=w(L)=0$ yield that $C=D=0$.

Consequently, since all the integration constants are zero the, case $k-m \omega^{2}=0$, yields no admissible deformation shape for the beam along the transverse direction; and therefore, the frequency value, $\omega=\sqrt{k / m}$, cannot be an eigenvalue along the transverse direction.

Case 1: $k-m \omega^{2}>0$

For $k-m \omega^{2}>0$ the solution of (2) is well known to the literature (Timoshenko et al. [5], Ugural \& Fenster [6] among others), 


$$
w(z)=e^{\lambda z}(A \cos \lambda z+B \sin \lambda z)+e^{-\lambda z}(C \cos \lambda z+D \sin \lambda z),
$$

where

$$
\lambda^{4}=\frac{k-m \omega^{2}}{E I}>0
$$

With the aforementioned boundary conditions for flexure along the transverse direction $w(0)=d^{2} w(0) / d z^{2}=w(L)=d^{2} w(L) / d z^{2}=0$, the eigenvalues of the system are obtained from the solution of the homogeneous system

$$
\left[\begin{array}{cccc}
1 & 0 & 1 & 0 \\
0 & 1 & 0 & -1 \\
e^{\lambda l} \cos \lambda L & e^{\lambda l} \sin \lambda L & e^{-\lambda l} \cos \lambda L & e^{-\lambda l} \sin \lambda L \\
-e^{\lambda l} \sin \lambda L & e^{\lambda l} \cos \lambda L & e^{-\lambda l} \sin \lambda L & -e^{-\lambda l} \cos \lambda L
\end{array}\right]\left\{\begin{array}{l}
A \\
B \\
C \\
D
\end{array}\right\}=\left\{\begin{array}{l}
0 \\
0 \\
0 \\
0
\end{array}\right\}
$$

The solution of the associated characteristic equation is given by:

$$
\cos (2 \lambda L)=\cosh (2 \lambda L)
$$

Equation (9) can be satisfied only when $\lambda L=0$ (see graphs on Figure 6) which implies that $\lambda=0$; therefore, from Equation (7), $k-m \omega^{2}=0$-a finding that contradicts the initial assumption of this case, that $k-m \omega^{2}>0$. The above analysis shows that when the frequency is kept low enough so that $k-m \omega^{2}>0$, then this low frequency range does not contain any eigenvalues of the structure along the transverse direction. Accordingly, the eigenvalues of the system belong in the range $k-m \omega^{2}<0$.

Case 2: $k-m \omega^{2}<0$

It is well known in the literature (Timoshenko et al. [5], Clough \& Penzien [7] among others) that the eigenvalues of the homogeneous equation given by (2) for $k-m \omega^{2}<0$ are given by the expression

$$
\omega_{T n}=\sqrt{\frac{k}{m}+n^{4} \pi^{4} \frac{E I}{m L^{4}}}, n \in\{1,2, \ldots\}
$$

Equation (10) shows that the lowest transverse eigenfrequency $(n=1)$ of the isolated deck is $\omega_{T 1}=\sqrt{k / m+\pi^{4} E I / m L^{4}}$, therefore, it will be always longer than the first longitudinal frequency $\omega_{L 1}=\sqrt{k / m}$. Consequently, the limiting case model which idealizes the isolated deck on distinct bearings with a flexural beam with continuously distributed springs yields that, no matter how long the bridge is, the first transverse isolated period is always smaller than the isolated longitudinal period due to the flexural rigidity of the deck $\left(\pi^{2} \sqrt{E I / m L^{4}}\right)$. 


\section{LONGITUDINAL AND TRANSVERSE EIGENVALUES OF A BEAM WITH A SINGLE LONGITUDINAL AND TRANSVERSE SPRING AT THE MID-SPAN}

We now proceed with the eiganvalue analysis of the other limiting mechanical idealization -that of a beam where its transverse motion is isolated with springs at the mid-span and restrained at the end supports; while the longitudinal motion is isolated with identical elastic springs at all three supports (see Figure 5).

\section{Transverse Periods}

Given the symmetry of the problem we can analyze half of the beam with $l=L / 2$ where the right free end has zero slope $(d w(l) / d x=0)$ and the shear equals to the spring reaction. Note that this model yields only the odd eigenvalues.

The solution of the vibration of a beam with flexural rigidity, $E I$ and distributed mass, $m$ is (Timoshenko et al. [5], Clough and Penzien [7]),

$$
w(x)=A \sin \lambda x+B \cos \lambda x+C \sinh \lambda x+D \cosh \lambda x
$$

where now

$$
\lambda=\sqrt[4]{\frac{m \omega^{2}}{E I}}>0
$$

The boundary conditions of this configuration for vibrations along the transverse directions are zero translation and zero moment at the left end-support $\left(w(0)=d^{2} w(0) / d x^{2}=0\right)$, while at the right end $(x=l=L / 2) d w(l) / d x=0$ and $V(l)=-E I d^{3} w(l) / d x^{3}=-k / 2 w(l)=0$, where $k$ is the transverse stiffness of the bearing(s) at the mid-span.

With the abovementioned boundary conditions the eigenvalues of the system for vibrations along the transverse direction are obtained from the solution of the homogeneous system.

$$
\left[\begin{array}{cccc}
0 & 1 & 0 & 1 \\
0 & -1 & 0 & 1 \\
\cos \lambda l & -\sin \lambda l & \cosh \lambda l & \sinh \lambda l \\
-\lambda^{3} \cos \lambda l-\widetilde{k} \sin \lambda l & \lambda^{3} \sin \lambda l-\widetilde{k} \cos \lambda l & \lambda^{3} \cosh \lambda l-\widetilde{k} \sinh \lambda l & \lambda^{3} \sinh \lambda l-\widetilde{k} \cosh \lambda l
\end{array}\right]\left\{\begin{array}{l}
A \\
B \\
C \\
D
\end{array}\right\}=\left\{\begin{array}{l}
0 \\
0 \\
0 \\
0
\end{array}\right\}
$$

in which $\widetilde{k}=k / 2 E I$. The solution of the associated characteristic equation gives

$$
2 \widetilde{k} \cosh \lambda l \cos \lambda l\left[\tanh \lambda l-\tan \lambda l-\frac{2 \lambda^{3}}{\widetilde{k}}\right]=0
$$

Equation (14) is satisfied either when $\cos \lambda l=0$ or when $\tanh \lambda l-\tan \lambda l-2 \lambda^{3} / \widetilde{k}=0$. The condition $\cos \lambda l=0$ corresponds to $\lambda l=(2 n+1)(\pi / 2)$, which gives the eigenvalues of the simple supported beam (without springs at the mid-span). For instance the first transverse period, $T_{T 1} S S$, of the simple supported beam with length $L$ is $(n=0)$.

$$
T_{T 1}^{S S}=\frac{2}{\pi} \sqrt{\frac{m L^{4}}{E I}}
$$


while the second modal period, $T_{T 2}{ }^{S S}=T_{T 1}{ }^{S S} / 4$. By setting the quantity in brackets in Equation (14) equal to zero, one obtains

$$
\tanh \lambda l=\tan \lambda l+\frac{4(\lambda l)^{3}}{\xi}
$$

where $\xi=k L^{3} / 8 E I$ is a dimensionless parameter which expresses the relative contribution of the spring at the mid-span to the transverse flexural rigidity of the deck.

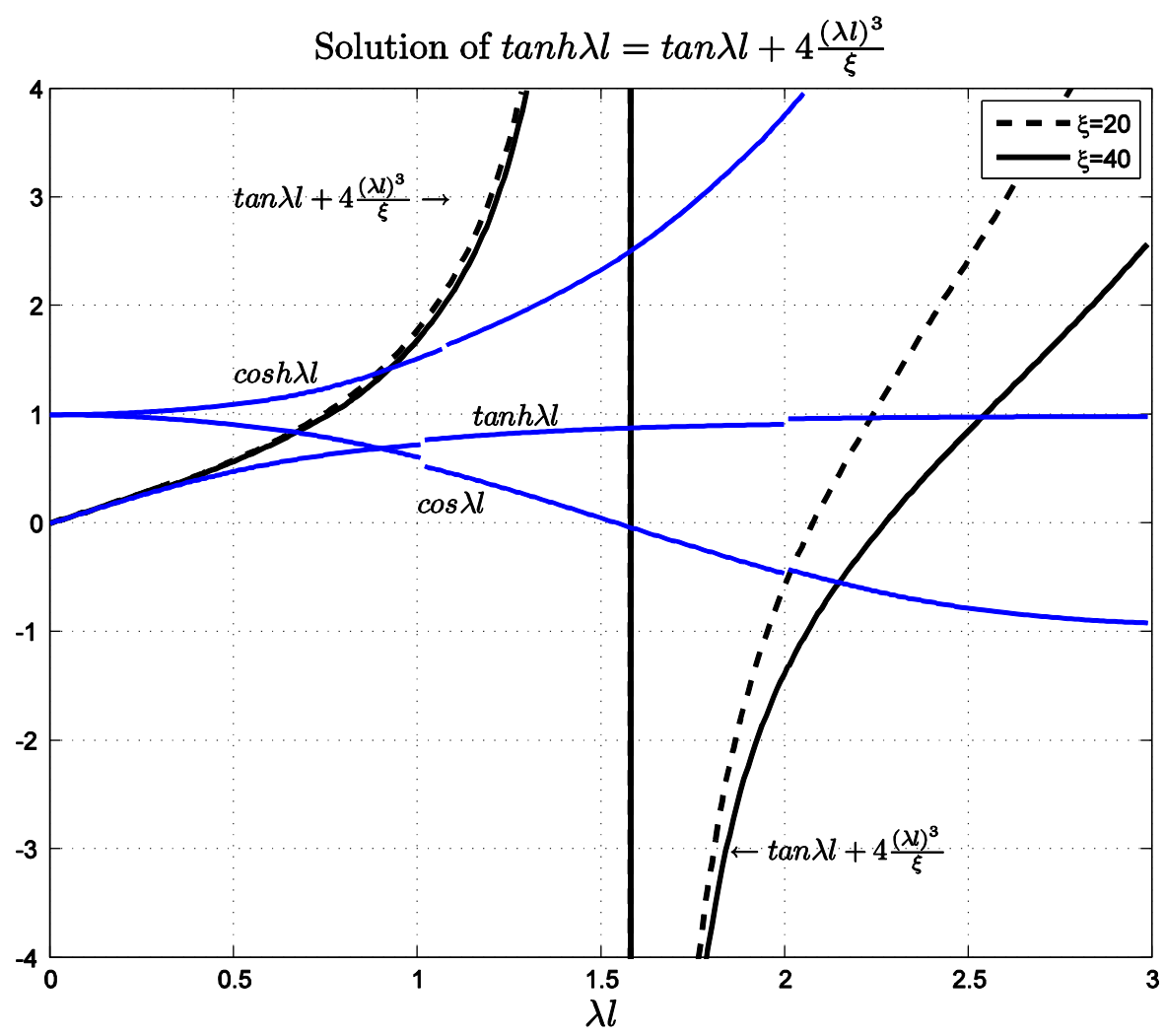

Figure 6. Graphical solution of the transcendental Equation (16).

The solution of the transcendental equation given by (16) is obtained for various values of $\xi$ either numerically with a Newton-Raphson method or graphically with the Creskoff diagram shown in Figure 6.

We first examine the solution of (16) at the limit of small $\xi$ (very soft spring or very rigid deck). Figure 7 plots the left-hand and the right-hand side of Equation (16) for $\xi=0.01$ and shows that there is a solution for $\lambda l=\pi / 2$; which is the solution of the first eigenvalue of the simple supported beam with length $L=2 l$ (see Equation (15)).

At the other limit of large $\xi$ (very stiff spring or very flexible deck) Equation (16) reduces to

$$
\tanh \lambda l=\tan \lambda l
$$


which is the characteristic equation of a beam with length $l=L / 2$ and with one end simple supported while the other fixed. In this case the first root is for $\lambda l=(1+1 / 4) \pi=5 \pi / 4$ and the first modal period of this configuration is

$$
T_{T 1}^{S F(L / 2)}=\frac{8}{25 \pi} \sqrt{\frac{m L^{4}}{E I}}=\frac{4}{25} T_{T 1}^{S S}
$$

Accordingly, as the stiffness of the bearing at the mid-span increases the transverse period of the bridge goes from the period of the simple supported beam with length $L=2 l$ given by Equation (15) to the period of the free-end-fixed-end beam with length $l=L / 2$ given by Equation (18). Figure 8 shows schematically the evolution of the mode shapes of the two-span beam as $\xi$ increases.

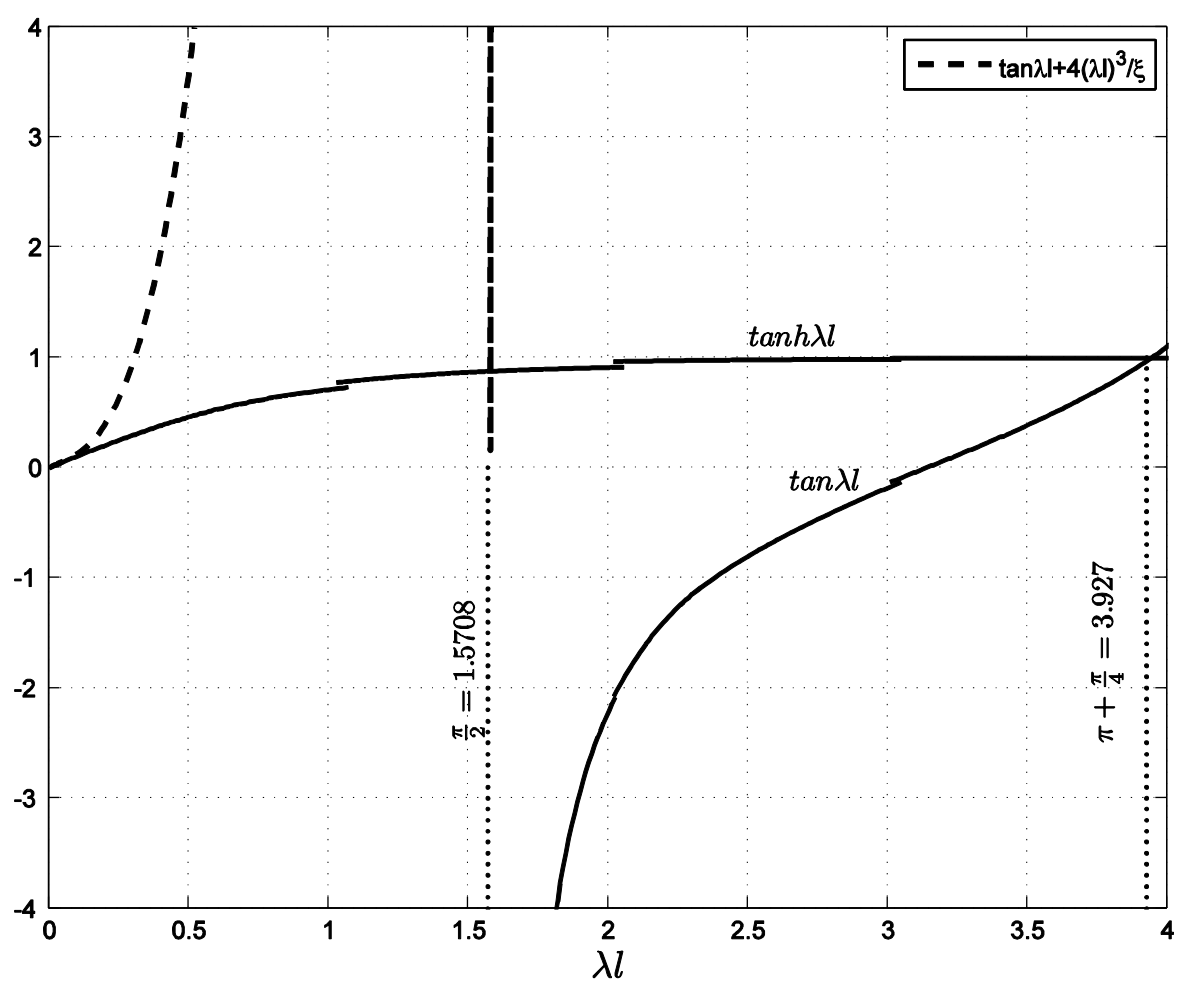

Figure 7. Behavior of the term $\tan \lambda l+4(\lambda l)^{3} / \xi$ at the limiting cases of small and large values of $\xi$.

We now return to Figure 6 where for finite values of, $\xi=k L^{3} / 8 E I$, the transcendental Equation (16) has real and positive solutions, say $S(\xi)=\lambda(\xi) l$ with $l=L / 2$ and $\lambda(\xi)=\sqrt[4]{m \omega^{2}(\xi) / E I}>0$. Accordingly,

$$
S(\xi)=\frac{L}{2} \sqrt[4]{\frac{m \omega_{T}^{2}(\xi)}{E I}}
$$

or 


$$
\omega_{T}(\xi)=4 S^{2}(\xi) \sqrt{\frac{E I}{m L^{4}}} .
$$

Equation (20) with the help of Equation (15) gives

$$
\frac{T_{T}}{T_{T 1} S S}=\frac{\pi^{2}}{4} \frac{1}{S^{2}(\xi)}
$$

in which, $T_{T}$, is the first transverse period of the two-span beam supported with a bearing at the mid-span. Figure 9 plots the expression given by Equation (21) after finding the roots of Equation (16), $S(\xi)=\lambda(\xi) l$, for the entire range of $\xi$ that is of interest. Note that for $\xi \rightarrow 0$ the ratio, $T_{T} / T_{T 1} S S$, tends to one; while, as $\xi \rightarrow \infty$, $T_{T} / T_{T 1}{ }^{S S} \rightarrow 4 / 25=0.16$ as is expected from Equation (18).
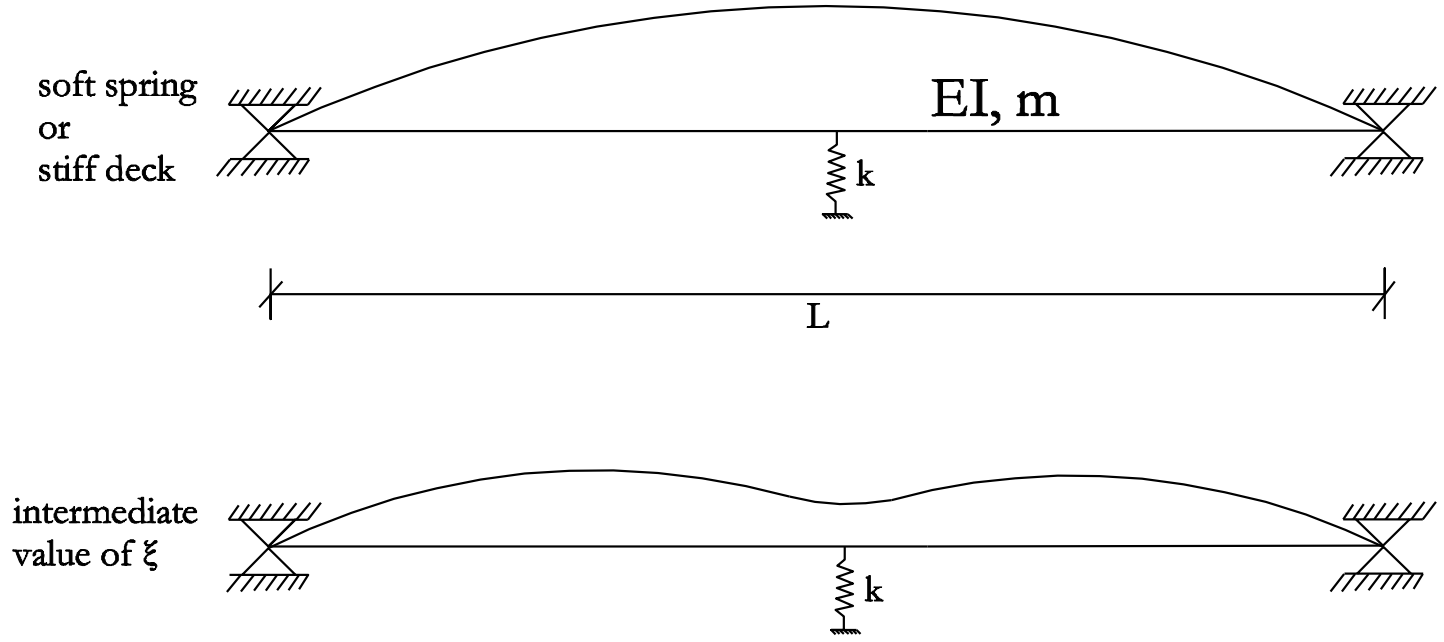

stiff spring or flexible deck
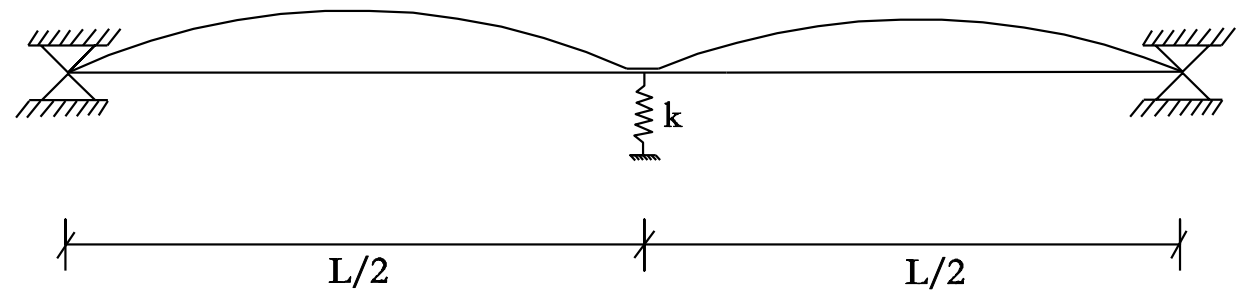

Figure 8. Schematic evolution of the transverse mode shape of a two-span beam as the stiffness of the center spring increases.

Figure 9 also shows that regardless whether the two-span deck is isolated on elastomeric or sliding bearings, the normalized transverse period of the two-span deck, $T_{T} / T_{T 1} S S$, is offered by a single master curve that is only a function of the normalized stiffness of the transverse spring at the mid-span $\xi=k L^{3} / 8 E I$.

This result can be also obtained qualitatively from dimensional analysis without solving the eigenvalue problem and the associated characteristic Equation (16). The 
transverse period of the two-span deck, $T_{T}[T]$ shall be only a function of the flexural rigidity of the beam, $E I[M][L]^{3}[T]^{-2}$, the distributed mass, $m[M][L]^{-1}$, the length of the beam, $L[L]$, and the stiffness of the transverse spring at mid-span $k[M][T]^{-2}$.

$$
T_{T}=f(E I, m, L, k)
$$

The five variables appearing in Equation (22) involve three reference dimensions, that of length $[L]$, time $[T]$ and mass $[M]$ and according to Buckingham's $\Pi$-theorem the number of independent dimensionless $\Pi$-products is equal to the number of physical variables appearing in Equation (22) (five variables) minus the number of reference dimensions (two). Accordingly, $5-3=2 \quad \Pi$-terms.

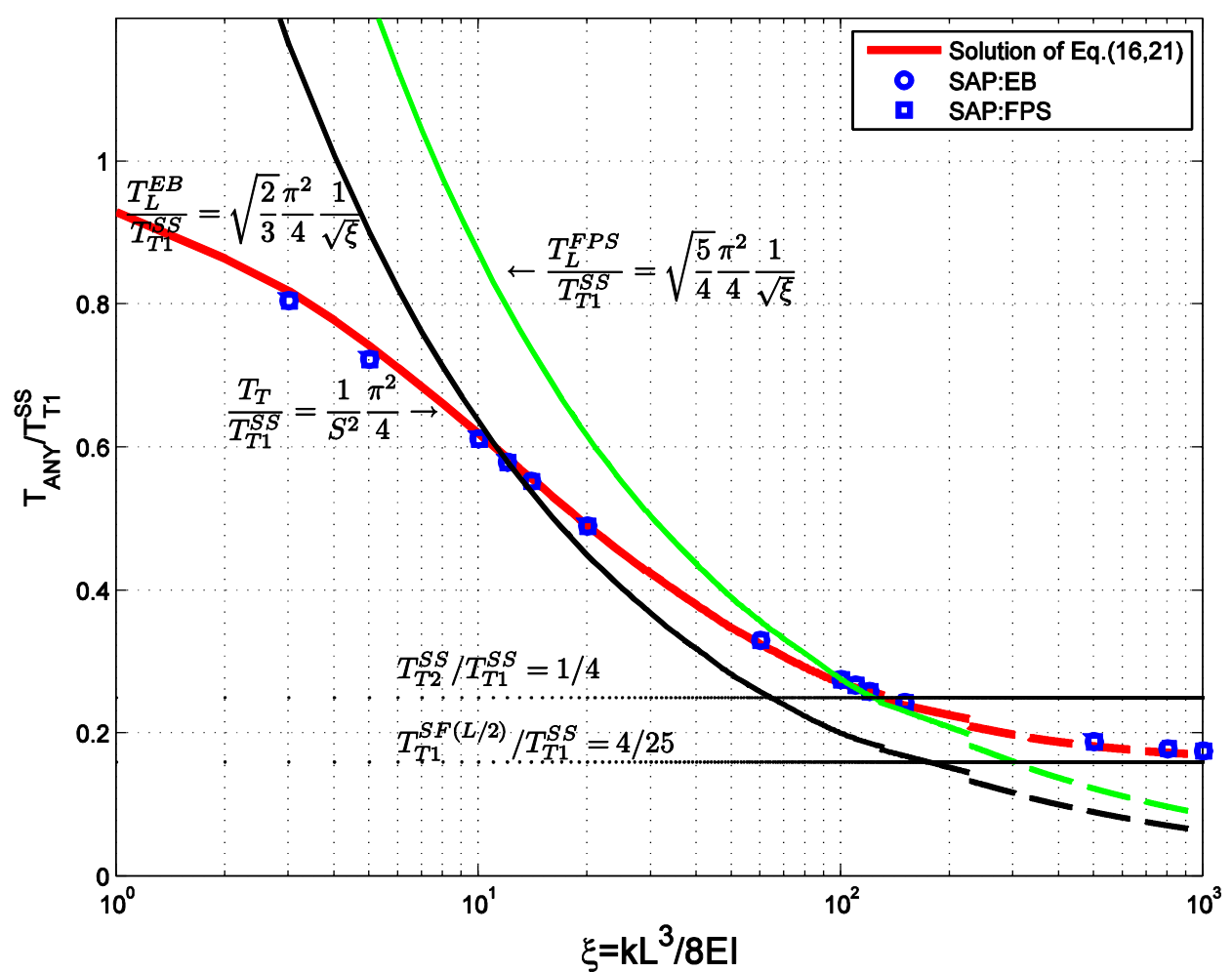

Figure 9. Comparison of the normalized first transverse period $T_{T}$ of a two-span isolated deck of length $L$ (solution of Equation (16)) against the longitudinal isolation periods $T_{L}^{E B}$ or $T_{L}^{F P S}$ ( EB =Elastomeric Bearings, FPS =Friction Pendulum System).

$$
\Pi_{1}=\frac{T_{T}}{\sqrt{m L^{4} / E I}}=\varphi\left(\frac{k L^{3}}{E I}\right)=\varphi\left(\Pi_{2}\right)
$$

Note that the denominator in the left hand side of Equation (23) is within a constant $(2 / \pi)$, the first modal period of the simple supported beam given by Equation (15); whereas, the independent, dimensionless $\Pi_{2}=k L^{3} / E I$ variable is within a constant (1/8) equal to $\xi$. The structure of Equation (23) dictated the synthesis of Figure 9 as well as the presentation of results regarding the eigenperiods of multi-span bridges in 
Figure 11. The numerical solution of the transcendental Equation (16) (heavy dark line in Figure 9) serves to validate the computations of the commercially available software, SAP (Computers and Structures [8]), which yields the point-circles when elastomeric bearings are used and the point-squares when friction pendulum bearings are used.

\section{Longitudinal Periods}

\section{a) Elastomeric Bearings}

When the two-span deck of Figure 5 is supported at each of the three supports (end abutments and mid-span) on identical elastomeric bearings with lateral stiffness $k$, the longitudinal period of the bridge is

$$
T_{L}^{E B}=2 \pi \sqrt{\frac{m L}{3 k}}
$$

The dimensionless stiffness of the bearing, $\xi=k L^{3} / 8 E I$, appearing in the righthand side of the characteristic Equation (16) gives

$$
k=\frac{8 \xi E I}{L^{3}}
$$

Substitution of Equation (25) to (24) gives,

$$
T_{L}{ }^{E B}=\frac{1}{\sqrt{6}} \frac{1}{\sqrt{\xi}} \frac{\pi}{2} \sqrt{\frac{m L^{4}}{E I}}
$$

From Equation (15) the first transverse period of the simple supported beam with length $L$ is $T_{T 1}^{S S}=\frac{2}{\pi} \sqrt{m L^{4} / E I}$, and therefore Equation (26) gives

$$
\frac{T_{L}^{E B}}{T_{T 1}^{S S}}=\sqrt{\frac{2}{3}} \frac{\pi^{2}}{4} \frac{1}{\sqrt{\xi}}
$$

Figure 9 plots the expressions given from Equation (27) next to the line given by Equation (23). Note that the two lines cross at an approximate value of $\xi \approx 12$, beyond which the transverse period of the two-span bridge supported on elastomeric bearings exceeds the longitudinal isolation period, $T_{L}{ }^{E B}$, given by Equation (27). This result is quite remarkable given that the solution from the beam on elastic foundation (infinite bearings) does not predict any crossing (the transverse period of a beam on Wrinkler foundation is always shorter than the longitudinal period regardless the length of the deck: Equation (10)).

\section{b) Spherical-Sliding Bearings}

We consider now the alternative situation where the two-span bridge of Figure 5 is supported at each of the three supports (end-abutments and mid-span) on identical spherical sliding bearings with radius of curvature $R$. In this case the longitudinal period of the bridge is

$$
T_{L}^{F P S}=2 \pi \sqrt{\frac{R}{g}}
$$


Given that the two-span beam is a continuous beam, the vertical reaction at the center bearing is $N_{C}=5 / 8 \mathrm{mgL}$, while the vertical reaction at the end-bearings is $N_{e}=3 / 16 \mathrm{mgL}$. Accordingly, the lateral stiffness of the center spherical sliding bearing is, $k=N_{C} / R=5 / 8 \mathrm{mgL} / R$ and the dimensionless stiffness of the center bearing is

$$
\xi=\frac{k L^{3}}{8 E I}=\frac{5}{64} \frac{m g L^{4}}{R E I}
$$

Substitution in (29) the ratio $g / R$ from Equation (28) gives,

$$
\frac{T_{L}{ }^{F P S}}{T_{T 1} S S}=\sqrt{\frac{5}{4}} \frac{\pi^{2}}{4} \frac{1}{\sqrt{\xi}}
$$

Figure 9 also plots the expression given from Equation (30) next to the line given by Equation (21). Note now that the two lines cross at an approximate value of $\xi \approx 116$. This result shows that the transverse period of a two-span isolated bridge with spherical sliding bearings which is transversely restrained at the end abutments may exceed the longitudinal isolation period $T_{L}{ }^{F P S}=2 \pi \sqrt{R / g}$; however, this may happen when the two-span bridge is significantly longer than the corresponding length of the two-span bridge isolated on elastomeric bearing that offer the same longitudinal period.

This remarkable result shows that while a bridge designer may use either elastomeric or spherical sliding bearings to achieve a desirable isolation period along the longitudinal direction, the transverse period of the deck when spherical sliding bearings are used will be always shorter (stiffer configuration) than the transverse period offered by elastomeric bearings which give the same isolation period along the longitudinal direction.

\section{THE TRANSVERSE EIGENVALUES OF MULTI-SPAN ISOLATED BRIDGES RESTRAINED AT THE END-ABUTMENTS}

Our investigation proceeds with the analysis of a 4-span and 8-span isolated deck with transverse restrains at the end-abutments. Similarly to the mechanical idealization shown in Figure 3, the 4-span and 8-span isolated beam have transverse restrains only at the end abutments while they are fully isolated along the longitudinal direction. A series of eigenvalue analyses have been conducted for bridge decks with different values of length and $E_{c}=25000 \mathrm{MPa}, I=100 \mathrm{~m}^{4}, \mathrm{~m}=50 \mathrm{Mg} / \mathrm{m}$ and isolation periods along the longitudinal direction $T_{L} \approx 3.0 \mathrm{~s}$ and $T_{L} \approx 2.0 \mathrm{~s}$. Both elastomeric bearing and spherical sliding bearing ( $R=2.2 \mathrm{~m}$ for $T_{L} \approx 3.0 \mathrm{~s}$ and $R=1.0 \mathrm{~m}$ for $T_{L} \approx 2.0 s$ ) have been considered.

Figure 10 (top) plots the first transverse period, $T_{T 1}$, of the 4-span deck normalized to the longitudinal isolation period, $T_{L}$ as a function of the length of the deck. For a 4span deck the longitudinal period is given either by

$$
T_{L}=T_{L}^{E B}=2 \pi \sqrt{\frac{m L}{5 k}}
$$


where $k$ is the lateral stiffness of the identical elastomeric bearings at each support, or by Equation (28) in which $R$ is the radius of curvature of any of the identical spherical sliding bearings. Regardless of the value of the isolation period, $T_{L}$ and whether the deck is isolated on elastomeric or spherical sliding bearings the ratio $T_{T 1} / T_{L}$ crosses the value one (1) in all cases; therefore, it is clear that in the case of a 4-span deck there are values of the length of the deck, $L$, beyond which the first transverse period, $T_{T 1}$, exceeds the longitudinal isolation period, $T_{L}$. Note that in the case of spherical sliding bearing (FPS) the first transverse period exceeds the longitudinal isolation period at deck lengths, $L$, appreciably longer than the length for which this happens when elastomeric bearings are used. This finding further confirms and generalizes the result discovered in Figure 9 (for a 2-span deck) that the transverse period of the deck when spherical sliding bearings are used will be always shorter (stiffer configuration) than the transverse period offered by elastomeric bearings which give the same isolation period (as the sliding bearing) along the longitudinal direction. Furthermore, note that this finding now has not only theoretical interest but also significant practical interest given the resulting span-lengths, $L / 4$, are of the order of $60 \mathrm{~m}$ to $75 \mathrm{~m}$ which are technically realizable.

Figure 10 (bottom) shows that the same phenomenon happens in the case of an 8-span deck. In the event that the 8-span deck is isolated at each of its nine (9) supports with elastomeric bearings having stiffness, $k$, the longitudinal period is given by $T_{L}=T_{L}{ }^{E B}=2 \pi \sqrt{m L / 9 k}$, while $T_{L}^{F P S}$ is given by Equation (28).

In this case three of the four crossings which happen in Figure 10 (bottom) occur for lengths of the 8-span deck $250 m<L<400 m$ which correspond to span-lengths from $L / 8=31.25 m$ to $L / 8=50 m$. Such span lengths are most common in prestressed isolated bridges in Greece and throughout the world.

The presentation of the computed transverse period of the 4-span and 8-span decks in Figure 10 as a function of the dimensional length, $L$, of the deck has practical interest since it offers directly for the given values of $E=25000 \mathrm{MPa}, I=100 \mathrm{~m}^{4}$ and $m=50 \mathrm{Mg} / m$ the values of length $L$ for which the ratio $T_{T 1} / T_{L}$ exceeds unity.

We are now interested to examine whether the four (4) distinct response curves shown at the top and bottom of Figure 10 are related to each other; or each one of them contains independent information. Whereas, the ratio $T_{T 1} / T_{L}$ is dimensionless it is not the appropriate dimensionless product to extract the physics of the problem. Equation (23) dictates that in order to uncover any existence of self-similar response the transverse period if the deck needs to be normalized to a time scale that reflects the flexural rigidity of the deck ( $E I / L^{3}$ ), while, the horizontal axis shall reflect the normalized lateral stiffness of the bearings $k$ at a single support to the flexural rigidity of the deck. Figure 11 (top) presents the same information as Figure 10 (top) in terms of dimensionless products $\Pi_{1}=T_{T 1} / T_{T 1} S S$ and $\Pi_{2}=\xi=k L^{3} / 8 E I$ where $T_{T 1}{ }^{S S}$ is given by Equation (15). Interestingly, the four distinct response curves appearing in Figure 9 (top) collapse to a single master curve shown in Figure 11 (top) 

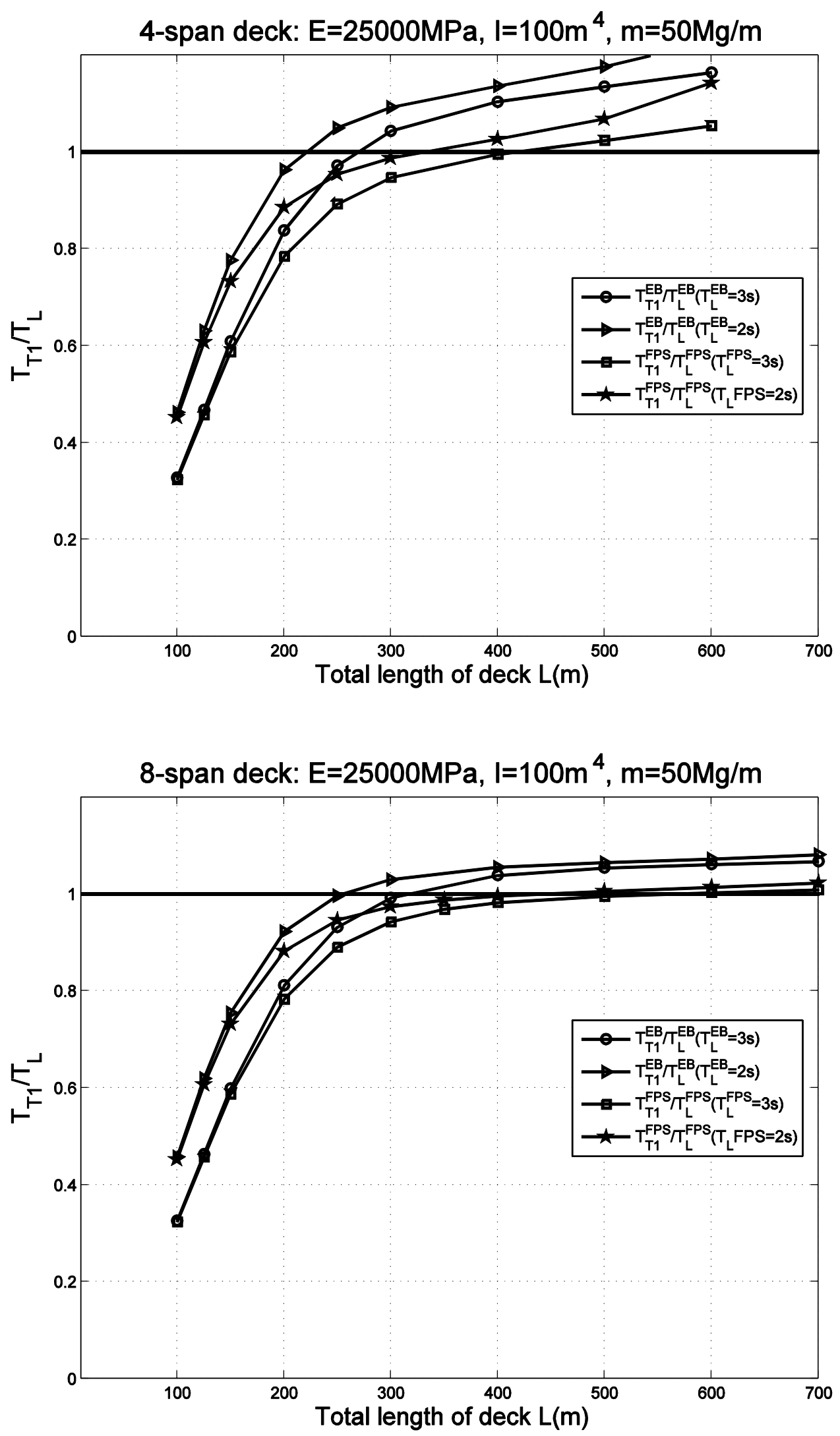

Figure 10. First transverse period of a deck isolated either with elastomeric or spherical sliding bearings normalized to the longitudinal isolation period $\left(T_{L}=2.0\right.$ or $\left.3.0 \mathrm{sec}\right)$.

Top: 4-span deck; Bottom: 8-span deck. 
which is independent of the longitudinal isolation period or whether the deck is supported on elastomeric or spherical sliding bearings. This single master curve, $T_{T 1} / T_{T 1} S S$ crosses the normalized longitudinal isolation periods either from elastomeric bearings $T_{L}^{E B} / T_{T 1}{ }^{S S}$ or from spherical sliding bearings $T_{L}{ }^{F P S} / T_{T 1}$ SS at two distinct values of $\xi$.

The same remarkable self-similar response emerges in Figure 10 (bottom) for the 8span deck. Note that the single master curve, $T_{T 1} / T_{T 1} S S$ also crosses the normalized longitudinal isolation periods $T_{L}^{E B} / T_{T 1}{ }^{S S}$ and $T_{L}{ }^{F P S} / T_{T 1} S S$; however, the intersection happens at a smaller angle than in the 4-span beam. Eventually as the number of spans increases, the master curve $T_{T 1} / T_{T 1} S S$ tends to become tangent to the curves representing the longitudinal isolation periods; and at the limit of infinite spans (beam on Winkler foundation) the curves will not cross as predicted by Equation (10).

Now, one shall notice that the self-similar response curves for the 4-span deck on Figure 11 (top) and the 8-span deck on Figure 11 (bottom), have also similar shapes.

Note that the horizontal axis in Figure 11 is $\Pi_{2}=\xi=k L^{3} / 8 E I$, that is the normalized lateral stiffness of the bearing(s) at a single support to the flexural rigidity of the deck. For any given value of $\xi=k L^{3} / 8 E I$, the 8-span deck enjoys the restoring transverse force from the bearings at seven (7) supports; while the 4-span deck enjoys the transverse restoring force from the bearings at only three (3) supports. We are now interested to express the transverse contribution of the bearing at all the supports of a multi-span bridge. Let assume for simplicity a bridge with an even number spans, $n=2 j$; and let approximate the first half-sine transverse mode shape of the deck with a triangle as shown in Figure 12. When the maximum amplitude of the mode shape is unity at the center of the deck the amplitude of the mode shape above the first pier next to the end abutment is approximately, $1 / j$, above the second pier is, $2 / j$, while, the amplitude of the deck above the pier before the center pier is, $(j-1) / j$.

Now, while the normalized transverse stiffness of the isolation bearings above every pier is $\xi$, the participation of the bearing stiffness at each location is proportional to the deformation that the bearing undergoes due to the prevailing half-sine mode shape of the deck. At the center of the deck where the amplitude of the mode shape $=1$, the participation is of the bearing above the center pier is $\xi$; while at any other location the participation is $(i / j) \xi,(i=1,2, \ldots, j-1)$. Accordingly, for a n-span bridge the transverse participation of all bearings from end abutments to end abutments is

$$
2 \frac{1}{j} \xi+2 \frac{2}{j} \xi+\ldots+2\left(\frac{j-1}{j}\right) \xi+\xi=\left[\frac{2}{j}(1+2+\ldots+(j-1))+1\right] \xi=j \xi=\frac{n}{2} \xi
$$

Given the result of the expression above Figure 13 plots the same information presented in Figure 11 (top and bottom) together with the results of Equation (21) (2span deck); where now the horizontal axis according to equation (32) is $j \xi=(n / 2) \xi=n k L^{3} / 16 E I$, where $n=$ number of spans. Interestingly all curves for the normalized transverse periods (2-span, 4-span and 8-span bridges) 

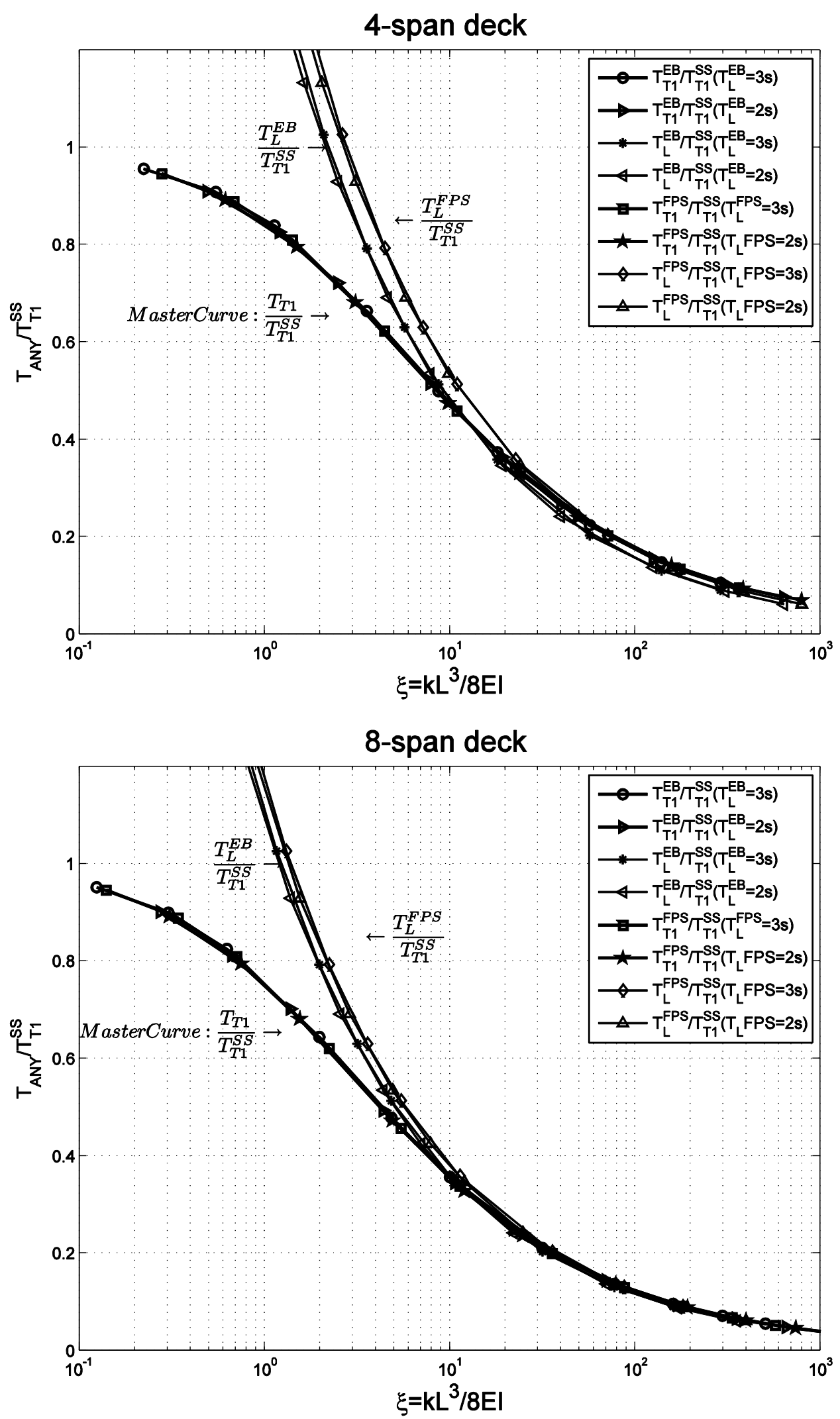

Figure 11. Comparison of the master curve of the first transverse period $T_{T 1}$ of an isolated deck with arbitrary longitudinal isolation period against the corresponding longitudinal isolation periods $T_{L}^{E B}$ or $T_{L}^{F P S}$ ( $E B=$ Elastomeric Bearings, FPS =Friction Pendulum System). Top: 4-span deck; Bottom: 8-span deck. 
collapse to a self-similar solution indicating that the first transverse eigenperiod of any multi-span isolated deck is given by a single master-curve. This remarkable result has significant practical value since the first transverse period of any finite-span isolated bridge with transverse restrains at the end abutments is merely offered with confidence from the solution of the Equation (16).

It is worth noting that beyond the value $(n / 2) \xi \approx 100$ the transverse period of the 2spn deck departs from the single master curve. This is because beyond $\xi=100(n=2)$ the spring at midspan is stiff enough so that the mode shape of the bridge departs appreciably from the half-sine and tends to the mode shape shown at the bottom of Figure 8 .

\section{$n$-span bridge $n=2 j$}
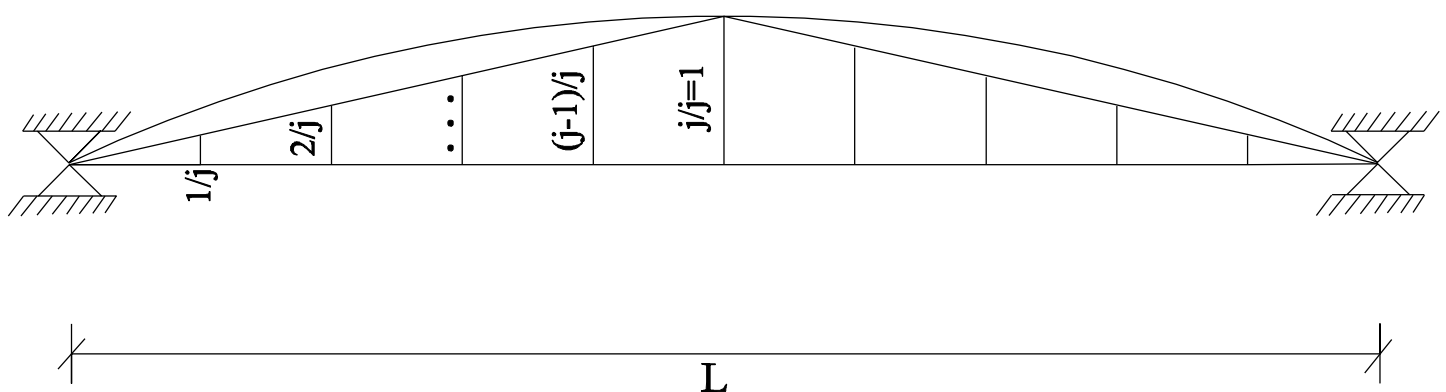

Figure 12. Approximation of the half-sine transverse mode shape of an isolated deck with a triangle together with the normalized transverse displacement of the bearings above the piers.

\section{CONCLUSIONS}

This paper examines the eigenvalues of relatively long seismically isolated bridges in which the transverse displacement of the deck at the end-abutments is restricted. With this restriction the deck is fully isolated along the longitudinal direction, while along the transverse direction the deck is a simple supported beam at the end-abutments which enjoys concentrated restoring forces from the isolation bearings above the center piers. This study first investigates mathematically the eigenvalue problem of a two-span isolated deck and subsequently examined numerically a 4-span and a 8-span isolated deck. The study concludes that regardless of the value of the isolation period along the longitudinal direction there is a certain length beyond which the transverse period of the deck will exceed the longitudinal isolation period. The value of this length depends on whether the deck is isolated on elastomeric or sliding bearings.

The transverse period of the deck when spherical sliding bearings are used will be always shorter (stiffer configuration) than the transverse period offered by elastomeric bearings which give the same isolation period along the longitudinal direction. Accordingly, this finding may influence the design of the deck in association with the selection of the type of isolation bearings in order to achieve the target transverse period. The above results were obtained only after considering local springs at the deck since the beam on distributed elastic supports (Beam on Wrinkler foundation) is unable to capture this phenomenon. Finally, using arguments from dimensional analysis the paper shows, that the normalized transverse eigenperiods of any finitespan deck are self-similar solutions which are independent of the longitudinal 


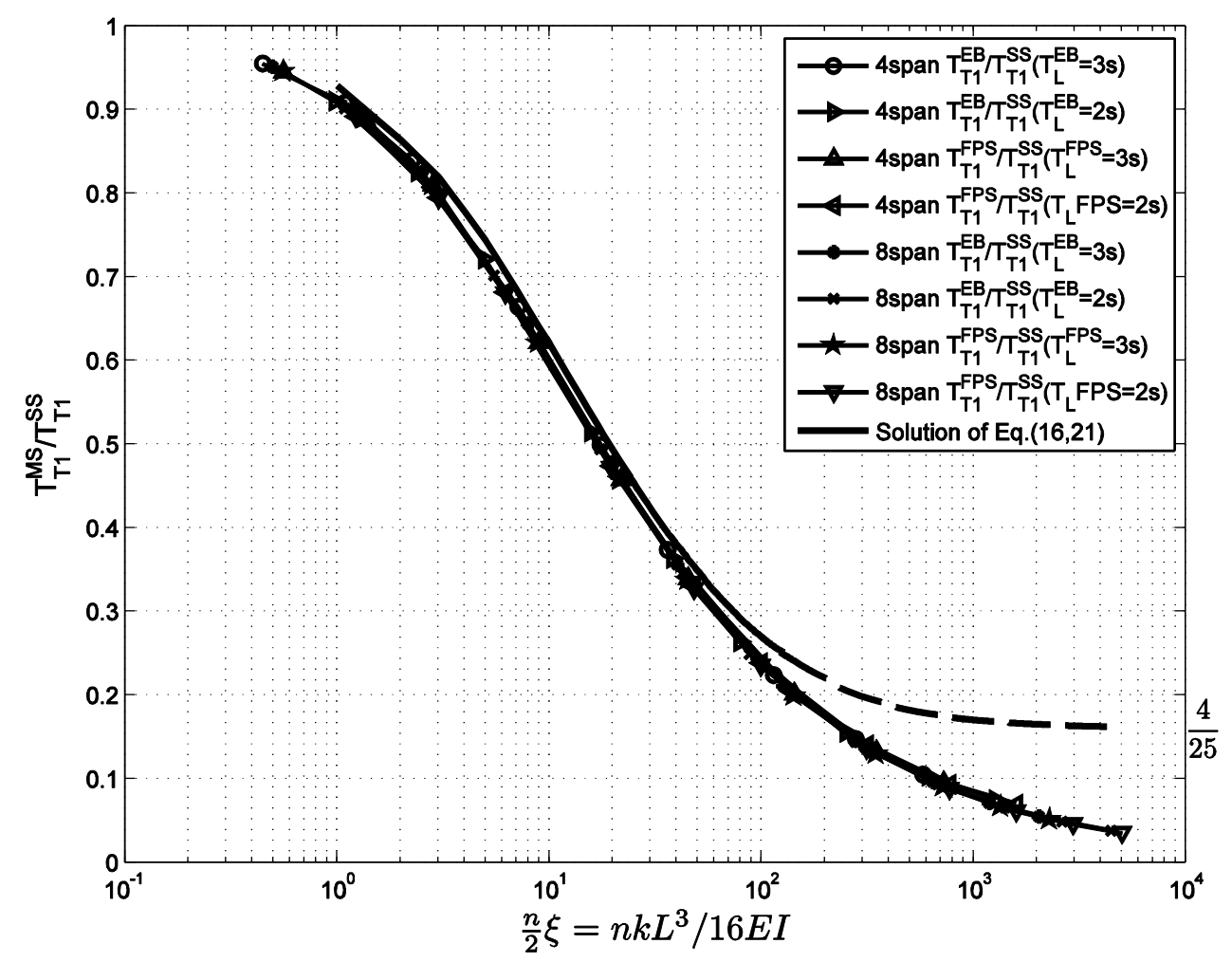

Figure 13. Master curve of the first transverse period of any multi-span isolated deck with arbitrary longitudinal isolation period.

isolation period of the deck or whether the deck is supported on elastomeric or spherical-sliding bearings. This remarkable self-similar behavior dictates that the first transverse period of any finite-span isolated deck with transverse restrains at the end abutments is merely offered from the analytical characteristic equation of the twospan beam.

\section{REFERENCES}

1. Skinner, R.I., Robinson, W.,H., McVerry, G., H. An Introduction to Seismic Isolation. Wiley, New York, 1993.

2. FHWA. Seismic Retrofitting manual for Highway Bridges. U.S. Department of Transportation: U.S.A., 1995.

3. Kelly, J., M. Earthquake-Resistant Design with Rubber. Springer-Verlag, London, 1997.

4. Zhang, J. and Makris N. Seismic Response Analysis of Highway Overcrossings Including Soil-Structure Interaction. Earthquake Engineering and Structural Dynamics 2002; 31:1967-1991.

5. Timoshenko, S., P., Young, D., H., Weaven, W., JR. Vibration Problems in Engineering ( ${ }^{\text {th }}$ Edition). Wiley, New York, 1974.

6. Ugural, A., C., Fenster, S., K. Advanced Strength and Applied Elasticity ( ${ }^{\text {rd }}$ Edition). Prentice-Hall, New Jersey, 1995.

7. Clough, R.W., Penzien, J. Dynamics of Structures. 2nd edition, McGrawHill, New York, 1993.

8. Computers and Structures. SAP 2000 Documentation. University of California, Berkeley, 2006. 